02

\title{
Оптические характеристики облученных гамма-лучами полимерных сцинтилляторов
}

\author{
() С.В. Афранасьев ${ }^{1}$, А.Ю. Бояринцев ${ }^{2}$, И.А. Голутвин ${ }^{1}$, Э.М. Ибрагимова ${ }^{3}$, А.И. Малахов ${ }^{1}$, И. Нуритдинов ${ }^{3, \uparrow, ~}$ \\ В.А. Смирнов ${ }^{1}$, М.Ю. Ташметов ${ }^{3}$, Б.С. Юлдашев ${ }^{3}$, З.У. Эсанов ${ }^{3}$ \\ ${ }^{1}$ Объединенный институт ядерных исследований, \\ 141980 Дубна, Россия \\ ${ }^{2}$ Институт сцинтилляционных материалов НАН, \\ 61000 Харьков, Украина \\ ${ }^{3}$ Институт ядерной фризики АН Узбекистана, \\ 100214 Ташкент, Узбекистан \\ P e-mail: izzatilloh@yahoo.com
}

Поступила в редакцию 25.02.2020 г.

В окончательной редакции 14.04.2020 г.

Принята к публикации 04.05.2020 г.

Исследовано влияние мощности дозы от 0.00022 до $0.0032 \mathrm{MGy} / \mathrm{h}$ и дозы облучения 0.043 и $0.2 \mathrm{MGy}$ гамма-источника ${ }^{60} \mathrm{Co}$, а также окислительных условий на оптические спектры пропускания и поглощения пластиковых сцинтилляторов марок BC-408 (Bicron, США), UPS-923A (Amcrys-H, Украина) и LHE (ОИЯИ, Россия) при $300 \mathrm{~K}$. Показано, что с увеличением дозы облучения уменьшается оптическое пропускание сцинтилляторов в диапазоне $400-850 \mathrm{~nm}$. Заметного влияния адсорбированного кислорода на индуцированные оптические потери при указанных условиях не было обнаружено.

Ключевые слова: полимерные сцинтилляторы, гамма-облучение, адсорбированный кислород.

DOI: $10.21883 /$ OS.2020.09.49860.58-20

\section{Введение}

Полимерные сцинтилляторы (ПС) широко используются в различных экспериментах в качестве детекторов частиц. Эти сцинтилляторы проще в изготовлении, обладают хорошей чувствительностью и быстродействием и, кроме этого, относительно дешевы (см., например, [1]). Тем не менее под воздействием ионизирующих излучений постепенно ухудшаются оптические свойства сцинтилляторов и тем самым уменьшается его светоотдача [1]. В экспериментах, проводимых, например, на коллайдерах, требуются большие светимости, т.е. детекторы, использующие сцинтилляторы, должны выдерживать высокие дозы излучения (например, от 0.01 до 0.1 MGy в год в течение 10 лет). Таким образом, существует необходимость найти такие полимерные сцинтилляторы, которые могут выдерживать воздействие больших потоков частиц и высокие дозы облучения без существенного ухудшения их оптических свойств.

Некоторые исследования воздействия гамма-излучения на различные характеристики сцинтилляторов проводились и ранее (см., например, [2-10]). Так, в работах [2,3] предполагается, что в результате воздействия гамма-излучения относительно высоких доз (0.005 MGy/h или выше) деградация светового потока будут такой же, как и при более низких дозах. Авторы [4] ставят под сомнение это предположение, считая, что деградация механических свойств полимерных сцинтилляторов зависит от дозы облучения.
Некоторые исследователи указывают на сильную зависимость светового выхода сцинтилляторов от мощности дозы [1,5-7], что объясняют вхождением кислорода в объем сцинтилляторов при облучении: считают, что при наличии растворенного кислорода в ПС происходит более эффективное образование устойчивых центров окраски (не отжигающихся со временем), в то время как другие утверждают, что значимой зависимости от мощности дозы нет [8].

Таким образом, на сегодняшний день не существует однозначного мнения о влиянии мощности и интегральной дозы облучения, а также вхождения кислорода в изменения характеристик полимерных сцинтилляторов при облучении.

В настоящей работе исследовано влияние мощности и дозы гамма-облучения, а также присутствия кислорода на спектры светопропускания и оптического поглощения облученных ПС марок BC-408, UPS-923A и LHE.

\section{Экспериментальные методы}

Спектры пропускания света и оптического поглощения ПС измерялись в диапазоне 200-800 nm на спектрофотометре (M40-Carl-Zeiss), а также в диапазоне 190-1100 nm - на спектрофотометрах Lamda 35 (Perkin Elmer, США) и СФ 50 (ЛОМО, Россия). Для измерения спектров использованы образцы в виде пластинок размерами $25 \times 25 \times 4 \mathrm{~mm}^{3}$. Данный эксперимент проводился на гамма-установке Института ядерной 

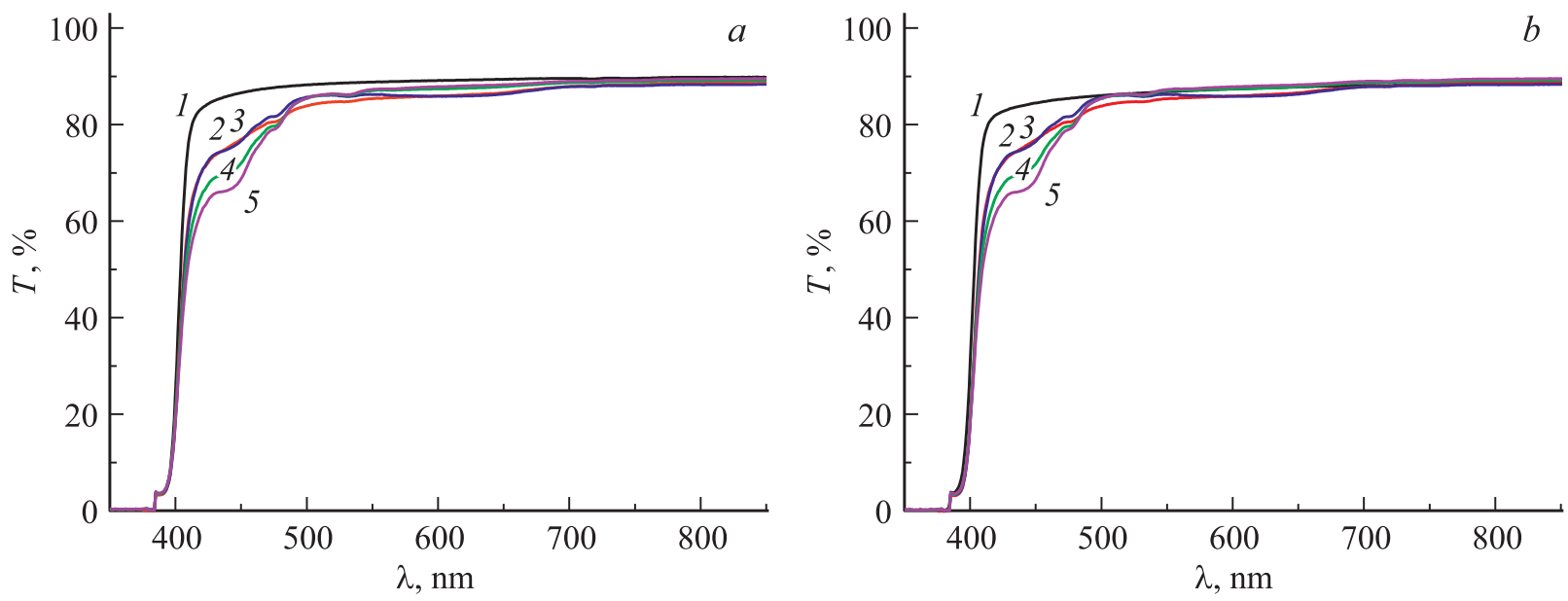

Рис. 1. Спектры пропускания образцов Bicron $(a)$ и UPS-923A $(b)$ : необлученные образцы $(1)$ и гамма-облученые на воздухе при $300 \mathrm{~K}$ до дозы $0.043 \mathrm{MGy}$ при мощностях: 0.00022 (2); 0.00044 (3), 0.0019 (4) и $0.0032 \mathrm{MGy} / \mathrm{h}$ (5).

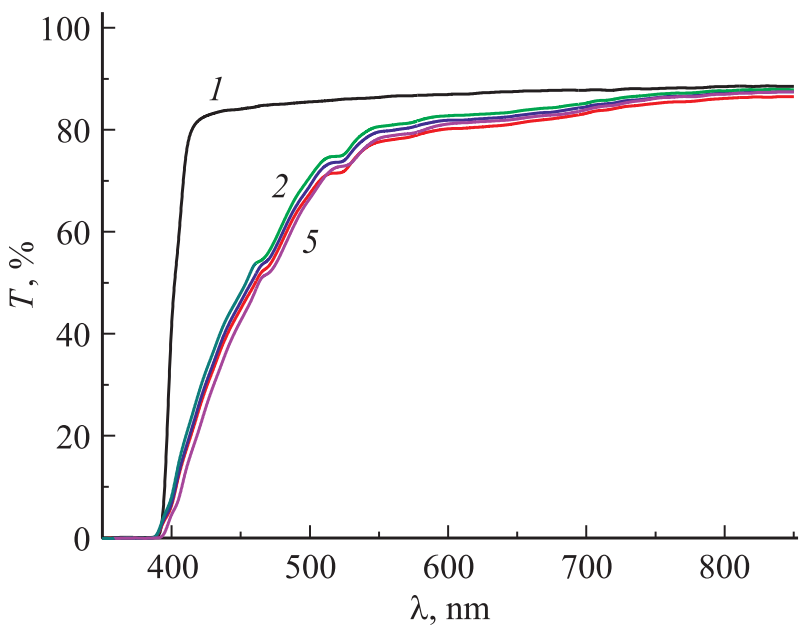

Рис. 2. Мощностная зависимость спектров пропускания образцов LHE до облучения (1) и измеренные сразу после облучения дозой $0.2 \mathrm{MGy} \mathrm{в} \mathrm{герметичной} \mathrm{упаковке} \mathrm{мощностями} 0.0019$ (2), 0.0032 (3), 0.0059 (4), $0.019 \mathrm{MGy} / \mathrm{h}$ (5).
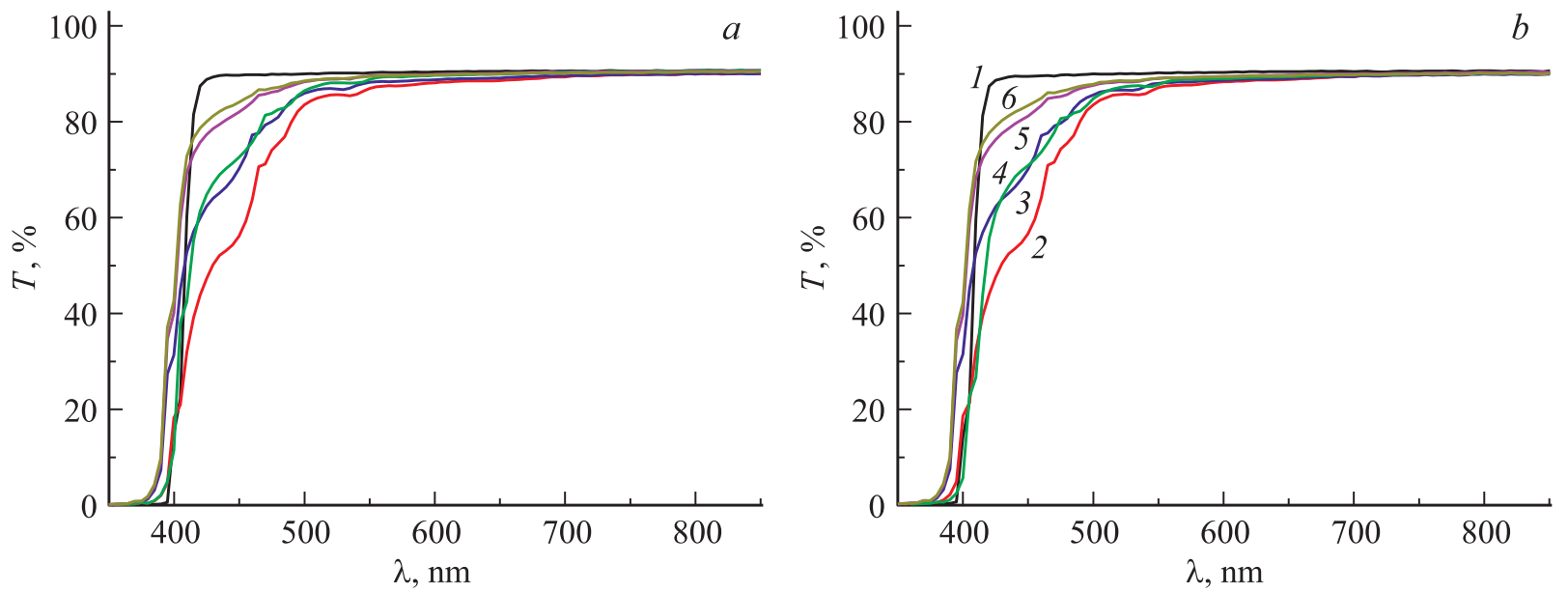

Рис. 3. Спектры пропускания образцов Bicron-408 до облучения (1), сразу после гамма-облучения дозой 0.2 MGy при мощности $0.019 \mathrm{MGy} / \mathrm{h}(2)$ и после релаксации через 1 день (3), 2 дня (4), 4 дня (5), 5 дней (6) герметично облученных (a) и облученных в контакте с воздухом $(b)$. 

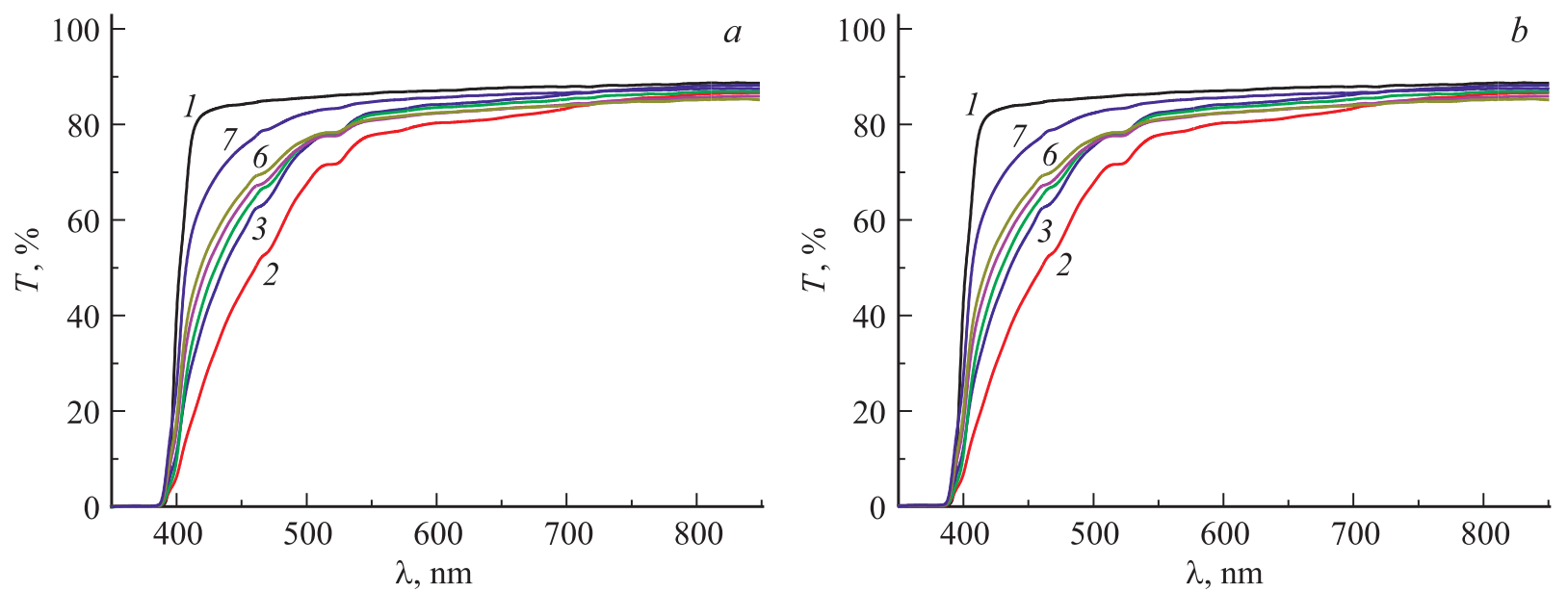

Pис. 4. Спектры пропускания образцов LHE в герметичной упаковке $(a)$ и в контакте с воздухом $(b)$ до облучения $(1)$, сразу после гамма-облучения при мощности $0.0019 \mathrm{MGy} / \mathrm{h}$ интегральной дозой $0.20 \mathrm{MGy}(2)$, через 1 день (3), 2 дня (4), 3 дня (5), 4 дня (6), 7 дней (7).
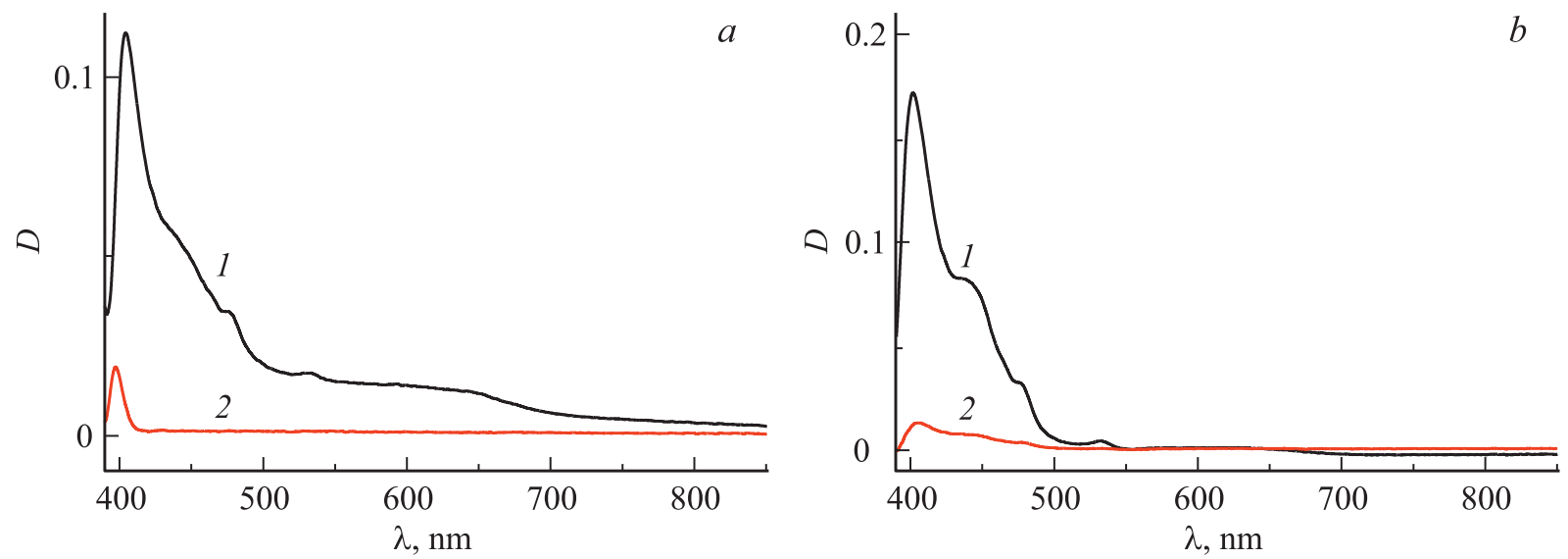

Рис. 5. Спектр наведенного поглощения образцов Bicron-408 сразу после гамма-облучения дозой $0.043 \mathrm{MGy}(1)$ и через 5 дней после облучения (2), облученных при мощностях $0.00022 \mathrm{MGy} / \mathrm{h}(a)$ и $0.0019 \mathrm{MGy} / \mathrm{h}(b)$.

физики АН Республики Узбекистан. Образцы облучались гамма-лучами источника ${ }^{60} \mathrm{Co}$ в гамма-установке бассейнового типа при мощностях $0.00022,0.00044$, 0.0019 и $0.0032 \mathrm{MGy} / \mathrm{h}$ интегральными дозами 0.043 и $0.2 \mathrm{MGy}$ при температуре канала $\left(30-50^{\circ} \mathrm{C}\right)$ в воздушной среде. Доза гамма-облучения определялась по изменению оптической плотности пленочного радиохромного детектора B3002 (Gex Corporation, США), измеренной с помощью спектрофотометра Thermo Genesys 20 (Gex Corporation, США), а мощность дозы определялась по изменению оптической плотности в единицу времени. Ошибка дозиметрии составляет около $10 \%$.

Для выяснения роли кислорода в изменении характеристик гамма-облученных сцинтилляторов, половину образцов облучали в герметичной упаковке (алюминиевая фольга и клейкая лента марки $\left.\operatorname{Scotch}^{\circledR}\right)$, чтобы устранить взаимодействие поверхности образцов с кислородом воздуха, а другую неплотно заворачивали в алюминиевую фольгу. Кинетика релаксации образцов после облучения снималась каждые сутки в одно и то же время (образцы хранились в темноте). Из исследуемых образов каждой марки один выбран в качестве эталона, который не подвергался облучению и использовался далее для относительных измерений. Облученные образцы хранились в темноте, чтобы избежать фотоиндуцированного обесцвечивания.

\section{Результаты экспериментов и их обсуждение}

Для каждого образца сначала проводили исходные (до облучений) измерения спектров пропускания относительно воздуха всех исследуемых образцов. Во всех исследованных образцах наблюдается резкое увеличение пропускания в районе $400 \mathrm{~nm}$. Разброс пропускания образцов в интервале $400-800 \mathrm{~nm}$ не превышает $2 \%$ и 

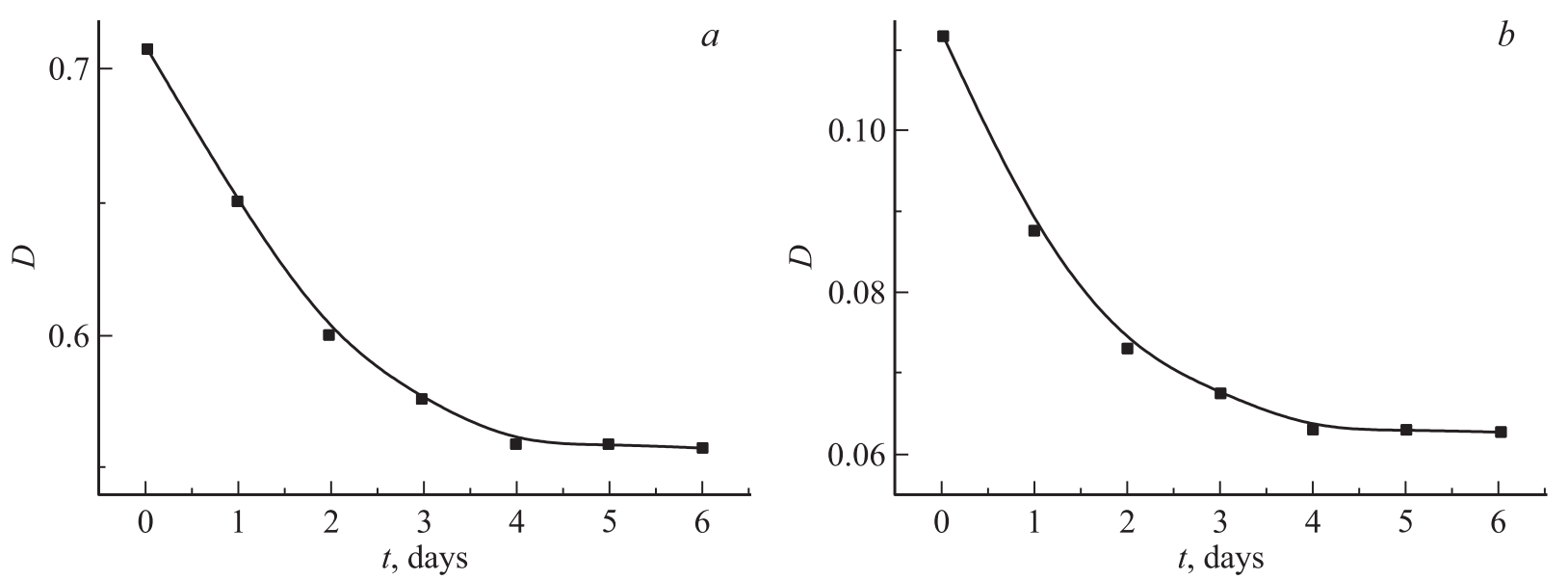

Рис. 6. Кинетика релаксации наведенного поглощения $\gamma$-облученного дозой $0.043 \mathrm{MGy}$ образца Bicron на полосе $450 \mathrm{~nm}$ при мощностях $0.000 .2 \mathrm{MGy} / \mathrm{h}(a)$ и $0.0019 \mathrm{MGy} / \mathrm{h}(b)$.
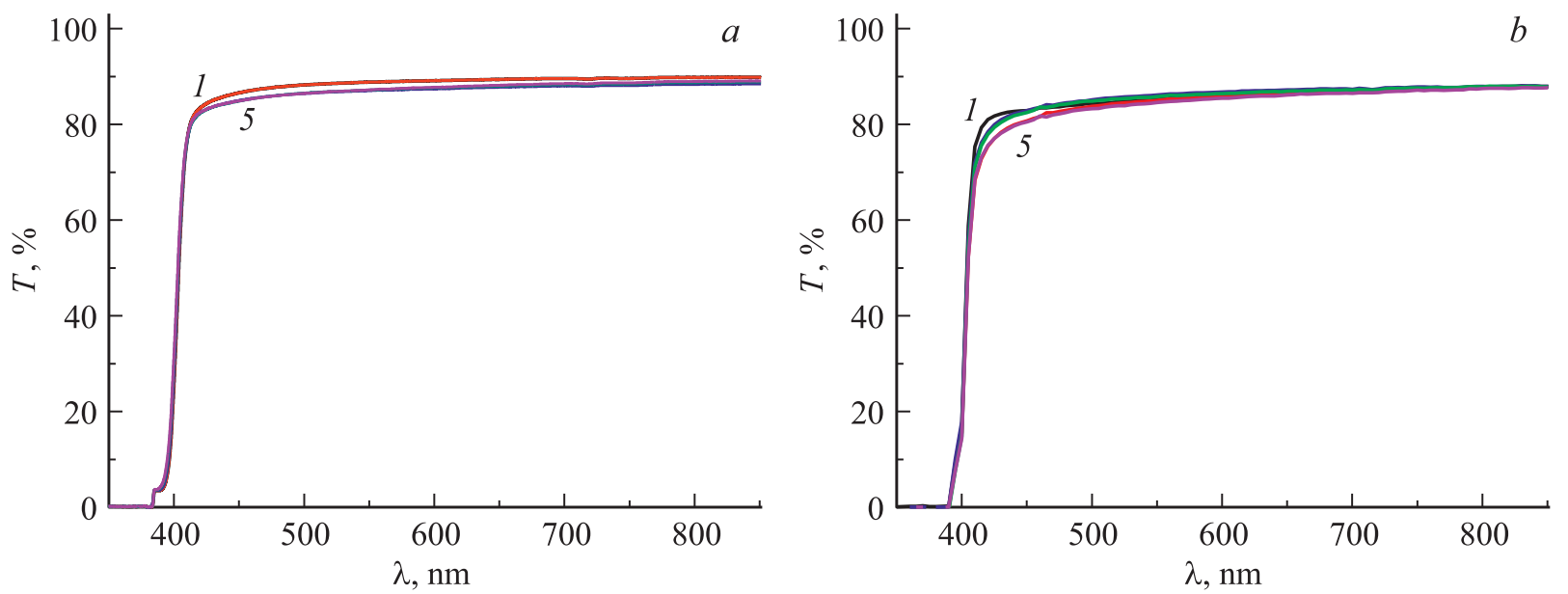

Pис. 7. Спектры пропускания необлученных $(1)$ и через 6 дней после завершения облучения образцов Bicron-408 (a) и UPS923A $(b)$, облученных дозой $0.043 \mathrm{MG}$, при мощностях дозы $0.000 .2(2), 0.00044$ (3), 0.0019 (4) и $0.0032 \mathrm{MGy/h}(5)$.

составляет $85-87 \%$. Поэтому в дальнейшем будут учитываться радиационно-индуцированные изменения оптического пропускания, превышающие $4 \%$ относительно необлученного образца.

На рис. 1-3 приведены спектры оптического пропускания необлученных и гамма-облученных интегральной дозой 0.043 MGy образцов UPS-923A, LHE и ВC-408 соответственно при различных мощностях дозы. Видно, что при облучении происходит ухудшение пропускания в области 400-850 nm у всех образцов, причем значение потери пропускания увеличивается с увеличением длины волны излучения до 800-850 nm, далее в спектрах не наблюдается изменений. Поэтому значение пропускания в спектрах всех измеренных образцов нормированы на $87 \%$ пропускания при длине волны $850 \mathrm{~nm}$. При одинаковых мощностях и дозах облучения минимальные потери наблюдаются у образцов Bicron, максимальные у образцов LHE.
При облучении образцов одинаковой марки с одинаковой интегральной дозой при различных мощностях облучения наблюдается увеличение потери пропускания с увеличением мощности дозы от 0.0022 до $0.0032 \mathrm{MGy} / \mathrm{h}$ (рис. 1), при более высоких мощностях до $0.019 \mathrm{MGy} / \mathrm{h}$ изменения очень слабые (рис. 2). Причиной этого может быть то, что чем меньше мощность дозы и интегральная доза облучения, тем меньше плотность наведенных дефектов в образцах. Мощностная зависимость демонстрирует наличие конкуренции между скоростью генерации дефектов (плотность нестабильных дефектов) и темпом их релаксации (рекомбинация, миграция, перенос заряда и т.д.). По-видимому, при мощности дозы $0.0032 \mathrm{MGy} / \mathrm{h}$ происходит насыщение наведенными дефектами образцов и устанавливается динамическое равновесие между скоростями генерации и релаксации дефектов в ПС.

Для выяснения роли радиационно-стимулированной диффузии кислорода из воздуха в объем ПС были 
исследованы спектры всех типов образцов при облучении их в герметичных упаковках, где доступ кислорода практически невозможен, и в упаковках, где возможен доступ кислорода (рис. 3 и 4). Исследования показали, что в образцах не обнаруживается существенного отличия в спектрах образцов, облученных в герметичных и негерметичных условиях, т.е., согласно данным настоящего эксперимента, роль кислорода в образовании центров окраски в исследованных образцах несущественна.

На рис. 3 и 4 приведены спектры пропускания образцов марки LHE и Bicron-408 сразу после облучения дозой $0.2 \mathrm{MGy}$ при мощностях 0.0019 и $0.019 \mathrm{MGy} / \mathrm{h}$ и после их временной релаксации. Из данных видно, что с течением времени на всех исследованных типах ПС наведенная облучением окраска уменьшается, что обусловливает увеличение пропускания образцов в области 400-800 nm (рис. 3 и 4). Для других доз и мощностей наблюдаются аналогичные зависимости.

Релаксация наведенной окраски особенно отчетливо проявляется на спектрах поглощения образцов. На рис. 5 для примера приведены спектры наведенного облучением поглощения образцов Bicron-408 сразу после гаммаоблучения дозой $0.043 \mathrm{MGy}$ и через 5 дней после облучения при мощностях 0.00022 и $0.0019 \mathrm{MGy} / \mathrm{h}$.

Временны́е зависимости релаксации наведенного поглощения этих образцов на полосе $450 \mathrm{~nm}$ приведены на рис. 6. Как видно, релаксация поглощения близка к экспоненциальной. Однако спектры не восстанавливаются до состояния необлученных образцов (рис. 3 и 4). Причем значение невосстановленного пропускания при одинаковых дозах облучения увеличивается с увеличением мощности дозы облучения (рис. 7).

\section{Выводы}

Приведенные выше экспериментальные результаты позволяют сделать следующие выводы.

1. При облучении гамма-квантами пластиковых сцинтилляторов UPS-923A, LHE и BC-408 происходит ухудшение пропускания в области 400-850 nm, причем значение потери пропускания увеличивается с повышением дозы облучения.

2. При одинаковых мощностях и дозах облучения минимальные потери наблюдаются у образцов Bicron, максимальное потери - у образцов LHE. При облучении образцов с одинаковой интегральной дозой при различных мощностях облучения наблюдается увеличение потери пропускания с увеличением мощности лозы от 0.00022 до $0.0032 \mathrm{MGy} / \mathrm{h}$; при более высоких мощностях (до $0.019 \mathrm{MGy} / \mathrm{h}$ ) изменения очень слабые, что свидетельствует о наличии конкуренции между скоростью генерации дефектов (плотность нестабильных дефектов) и темпом их релаксации (рекомбинация, миграция, перенос заряда).
3. Влияние адсорбированного кислорода в образовании радиационных дефектов и потери пропускания в исследованных условиях облучения практически отсутствует.

4. С течением времени на всех типах сцинтилляторов наведенная облучением окраска уменьшается, закон релаксации дефектов близок к экспоненциальному. Однако спектры не восстанавливаются до состояния необлученных образцов, причем значение невосстановленного пропускания при одинаковых дозах облучения увеличивается с увеличением мощности дозы облучения.

\section{Список литературы}

[1] Levy P.W., Kierstead J.A. // IEEE Trans. Nucl. Sci. 1991. P.1493-1497.

[2] Dormenev V., Auffray E., Brinkmann K.-T., Korjik M., Lucchini M.T., Mechinsky V., Novotny R.W., Zaunick H.G. // Conference Record IEEE Nuclear Science Symposium MIC. France. 29 Oct. 6 November. 2016. P. 1608-1611. doi 10.1109/NSSMIC.2016.8069809

[3] Hasegawa T., Hazumi M., Kasai S., Tokushuku K., Yamada S., Kojima T. Shimizu T. // Nucl. Instr. and Meth. A. 1992. V. 311. P. 498-504. doi 10.1016/0168-9002(92)90647-M

[4] Wilski H. // Radiat. Phys. Chem. 1987. V. 29. P. 1-14. doi 10.1016/1359-0197(87)90054-3

[5] Tome B., Gomes A., Henriques A., Maio A., Peralta L. Pereira M., Rocha J., Sonderegger P. // Radiat. Phys. Chem. 1993. V. 41. P. $185-193$. doi 10.1016/0969-806X(93)90055-Y

[6] Kross B., Majewski S., Wojcik R., Zom C. // Radiat. Phys. Chem. 1993. V. 41. P. 195-204. doi 10.1016/0969-806X(93)90056-Z

[7] Senchishin V.G., Lebedev V.N., Hlapova N.P., Adadurov A.F. // Issues of Atom science and Techniques. Series. Physics of Radiation Damages and Radiation Material Sciences. 2005. V. 86. N 3. P. $160-163$.

[8] Giokaris N.D., Contreras M., Pla-Dalmau A., Zimmerman J. // Radiat. Phys. Chem. 1993. V. 41. P. 315-320. doi 10.1016/0969-806X(93)90069-7

[9] Afanasiev S.V., Boyarintsev A.Yu., Danilov M.V., Emeliantchik I.F., Ershov Yu.V., Golutvin I.A., Grinyov B.V., Ibragimova E.M., Levchuk L.G.,. Litomin A.V., Makankin A.M., Malakhov A.I., Moisenz P.V., Nuritdinov I., Popov V.F., Rusinov V.Yu., Shumeiko N.M., Smirnov V.A., Sorokin P.V., Tarkovskii E.I., Tashmetov M.Yu., Vasiliev S.E., Yuldashev B.S., Zamiatin N.I., Zhmurin P.N. // CMS Note. 2015. V. 2. P. $1-15$.

[10] Afanasiev S.V., Boyarintsev A.Yu., Danilov M.V., Emeliantchik I.F., Ershov Yu.V., Golutvin I.A., Grinyov B.V., Ibragimova E.M., Levchuk L.G., Litomin A.V, Makankin A.M., Malakhov A.I., Moisenz P.V., Nuritdinov I., Popov V.F., Rusinov V.Yu., Shumeiko N.M., Smirnov V.A., Sorokin P.V., Tarkovskii E.I., Tashmetov M.Yu., Vasiliev S.E., Yuldashev B.S., Zamiatin N.I., Zhmurin P.N. // Nuclear Instruments and Methods in Physics Research. 2016. A 818. P. 26-31. doi 10.1016/j.nima.2016.02.045 